\section{S09.3 THE IMPACT OF SCREENING ON CHLAMYDIA TRANSMISSION IN AUSTRALIA -- A MATHEMATICAL MODELLING STUDY}

${ }^{1}$ Ben Hui* ${ }^{2}{ }^{2}$ Hocking, ${ }^{3} \mathrm{~N}$ Low, ${ }^{1}$ David Regan*. ${ }^{1}$ The Kirby Institute, UNSW Australia, Sydney, Australia; ${ }^{2}$ Melbourne School of Population and Global Health, University of Melbourne; ${ }^{3}$ nnstitute of Social and Preventive Medicine, University of Bern

10.1136/sextrans-2015-052270.54

Background Repeat chlamydia infection following treatment is common among young people and is associated with increased risk of pelvic inflammatory disease. Retesting and providing treatment to infected partners can reduce the reinfection rate. Both strategies have their own merits and limitations. Here we present a comparison of the impact of these two strategies on population prevalence through the use of modelling.

Methods An individual-based model was developed to represent the transmission of chlamydia in a heterosexual population. A proportion of the modelled population is tested for chlamydia and treated annually under the testing coverage achieved in ACCEPt. We estimate the additional reduction in chlamydia prevalence achieved by retesting individuals who were infected with chlamydia and compare with the estimated reduction achieved by treating the partners of infected individuals.

Results Preliminary results suggest that retesting at the rate achieved in ACCEPt would yield an additional $0.7 \%$ to $1.0 \%$ reduction in chlamydia prevalence. This reduction is greater than would be achieved by treating $10 \%$ of infected partners of index cases alone, which only yields an additional $0.5 \%$ to $0.8 \%$ reduction in chlamydia prevalence. If $10 \%$ of infected partners are treated in addition to retesting, the model predicts a further $0.6 \%$ to $0.7 \%$ reduction in chlamydia prevalence.

Conclusion Both retesting and partner treatment can result in reductions in chlamydia prevalence in the population. Assuming that the retesting rate achieved in ACCEPt can be extended to the general Australian heterosexual population, this strategy should result in a greater reduction in prevalence than treating $10 \%$ of infected partners. More substantial reductions can be achieved by combining both strategies.

Disclosure of interest statement The Kirby Institute is funded by the Australian Government Department of Health and Ageing and is affiliated with the Faculty of Medicine, University of New South Wales. The views expressed in this publication do not necessarily represent the position of the Australian Government.

\section{S09.4 WHAT DOES ACCEPt MEAN FOR CHLAMYDIA CONTROL POLICY?}

Helen Ward*. Imperial College London, London, UK

10.1136/sextrans-2015-052270.55

Chlamydia infection is endemic among sexually active young adults. A recent systematic review of chlamydia prevalence in young adults in Europe and other high income countries estimated a pooled point prevalence of $3.6 \%(95 \%$ CI $2.4,4.8)$ in women and $3.5 \%(95 \%$ CI 1.9, 5.2) in men. These levels of infection have remained stubbornly persistent in many countries despite major investments in sexual health programmes. This leaves policy makers with a challenge: what can we do to control chlamydia?

Existing evidence underpinning chlamydia control policy comes from (a) observational studies showing the incidence of sequelae, (b) a small number of trials showing a reduction in
PID following a single offer of a chlamydia test to asymptomatic women, (c) ecological studies suggesting a decline in chlamydia following the introduction of widespread testing in some countries, (d) a small number of (inconclusive) studies the impact of testing interventions on population prevalence, (e) mathematical models estimating that screening programmes could have an impact on transmission and therefore prevalence if they achieve high coverage and repeated rounds of testing.

Overall there is a lack of a robust evidence base for the development of control policy.

The Australian Chlamydia Control Effectiveness Pilot (ACCEPt) promises to add to the evidence base by explicitly testing the effectiveness of an intervention in terms of changes in chlamydia prevalence. The results of the pilot will be discussed in relation to the wider evidence briefly described above.

\section{S10 - Promoting STI control policies and programmes post-2015: lessons for the future from Iran and China}

\section{S10.3 STI CONTROL IN CHINA: WHAT ACCOUNTS FOR POLITICAL PRIORITIES?}

Flora Dadong Wu*. Institute for Global Health, University College London, UK

\subsection{6/sextrans-2015-052270.56}

Introduction Despite a large and growing burden of mother-tochild transmission (MTCT) of syphilis in China over the past 20 years, the issue received far less attention and fewer resources than prevention of MTCT (PMTCT) of HIV, which has a substantially lower burden. China's Ministry of Health issued the first national plan for syphilis control in 2010, aiming to integrate PMTCT of syphilis and HIV. Our study aimed to identify: 1) why PMTCT of syphilis had a lower political/resource priority than PMTCT of HIV before 2010; and 2) what actions would improve the prospects of successful implementation of dual PMTCT.

Methods A comparative policy analysis was undertaken, based on informant interviews, documentation review, and nonparticipant observation of relevant meetings/trainings, to investigate priority-setting prior to 2010 . Policy data were analysed by using a nine-factor framework which assesses political prioritization across three categories: transnational influence; domestic advocacy; and national political environment.

Results Several factors contributing to the lower priority accorded to PMTCT of syphilis were identified: 1) relative neglect at a global level; 2) dearth of international financial and technical support; 3) poorly unified national policy community with weak accountability mechanisms; 4) insufficient understanding of the epidemic and policy options; and 5) a prevailing negative framing of syphilis that resulted in significant stigmatization. Conclusion The goal of dual PMTCT of syphilis and HIV will only be achieved when equal priority is accorded to both infections. This will require stronger cohesion and leadership from the syphilis policy community. The community will also need to reframe the issue so as to overcome stigmatization against those affected by the illness, organize focusing events to attract political attention, and work more closely with the HIV policy community in order to enhance the recognition of the need to control syphilis on both the national and sub-national agendas. 


\section{S11 - Molecular aspects of antimicrobial resistant Neisseria gonorrhoeae}

\section{S11.1 REAL-TIME PCR DETECTION OF N. GONORRHOEAE RESISTANCE: WHERE ARE WE NOW?}

David Whiley*. University of Queensland, Brisbane, Australia

10.1136/sextrans-2015-052270.57

Ongoing emergence and spread of Neisseria gonorrhoeae antimicrobial resistance is of global concern and was recently designated an "urgent threat" by the United States Centres for Disease Control and Prevention. Australia is not immune to the $N$. gonorrhoeae resistance problem as highlighted by the first report of a ceftriaxone-resistant strain from Australia in 2014. Enhancing antimicrobial resistance (AMR) surveillance strategies to advance detection of gonococcal AMR is a priority. Whilst bacterial culture-based methods remain the most definitive means of assessing N. gonorrhoeae AMR, there has been decreasing availability of cultured isolates for AMR susceptibility testing owing to increased use of nucleic acid amplification test (NAAT)-based methods for gonorrhoea diagnosis. Molecular AMR surveillance tools have the potential to overcome these problems. In particular, polymerase chain reaction (PCR)-based methods targeting key genetic markers of resistance can facilitate rapid, more intense sampling, for $N$. gonorrhoeae strains of public health importance following NAAT-based diagnosis. This presentation will discuss the development of real-time PCR methods for N. gonorrhoeae AMR detection and in doing so will highlight potential technical obstacles that may impinge upon assay design and performance.

\section{S11.2 EFFLUX PUMPS IN NEISSERIA GONORRHOEAE - CAUSE OF RESISTANCE AND TARGETS FOR THERAPEUTICS AND VACCINE?}

William Shafer*. Emory University School of Medicine, Atlanta, USA and Atlanta VA Medical Center, Atlanta, USA

\subsection{6/sextrans-2015-052270.58}

Neisseria gonorrhoeae is the cause of the sexually transmitted infection termed gonorrhoea, which afflicts over 100 million people worldwide each year. Since the mid-1940s, beginning with the availability of penicillin (Pen), antibiotic therapy has been the mainstay for curing infection and halting the spread of the gonococcus in the community. Unfortunately, gonococci developed resistance to Pen and many other antibiotics that were brought into clinical practice to counter act the growing problem of Pen (and other antibiotics) resistance. With the recent emergence of strains expressing resistance to the third-generation cephalosporins (cefixime and ceftriaxone) or other important antibiotics (macrolides and fluoroquinolones) there is considerable fear that without new antibiotics, gonorrhoea will become more difficult to treat; indeed, some have warned of the possibility of untreatable infections. In order to address this public health crisis of antibiotic resistant gonococci, it is essential that new bacterial targets are identified so as to facilitate the development of novel therapeutic drugs. I will discuss the role of the MtrC-MtrD-MtrE efflux pump in the development of gonococcal resistance to antibiotics and host-derived antimicrobials (e.g., cationic antimicrobial peptides). These phenotypes are augmented in gonococcal strains that have mutations that result in enhanced transcription of the mtrCDE efflux pump gene complex. I will provide evidence that this efflux pump is critical for the ability of gonococci to resist certain antibiotics as well as surviving during infection. Against this background, I will propose that this pump offers new targets for drug development (efflux pump inhibitors) and vaccine development to combat antibiotic resistant gonococcal strains and to prevent infections.

\section{S11.3 USE OF WHOLE GENOME SEQUENCING TO DETERMINE THE PROBABILITY OF ANTIMICROBIAL RESISTANCE IN NEISSERIA GONORRHOEAE}

David Trees*. Centers for Disease Control and Prevention, Atlanta, USA

\subsection{6/sextrans-2015-052270.59}

Neisseria gonorrhoeae has demonstrated remarkable ability to rapidly develop antimicrobial resistance to each antimicrobial class used for therapy including the currently recommended class of antimicrobials, the cephalosporins. Emerging resistance to cephalosporins will severely complicate treatment of gonorrhoea. Advanced Molecular Detection approaches, such as whole genome sequencing to determine mechanisms of resistance, have the potential to advance our understanding of gonorrhoea. To date, we have sequenced $>1,000$ genomes from $N$. gonorrhoeae isolates with elevated cefixime or azithromycin minimum inhibitory concentrations (MICs) or resistance to previously recommended antimicrobials, and isolates collected from multiple geographic sites to accomplish the following objectives: (1) to identify mutations that confer antimicrobial resistance or increased MICs; (2) to inform the development of molecular assays for resistance determinants through insights gleaned from WG from which point-of-care assays for resistance markers will be developed. Such assays will change the paradigm of gonorrhoea treatment. Use of real-time results on the presence or absence of resistance determinants will allow clinicians to personalise treatment for patients, and prevent inadvertent use of agents that are no longer routinely recommended because of resistance. Such assays can also substantially expand the reach of surveillance of antimicrobial resistance and allow public health officials to rapidly detect and respond to outbreaks of resistant strains; and (3) to develop and maintain a microbial library, a web-accessible and searchable database that will include bacterial and viral genomic sequences and associated meta-data, such as basic de-identified demographic characteristics of patients and phenotypic susceptibility data. In the long term, knowledge of the genetic mechanisms responsible for resistance or deceased susceptibility in N. gonorrhoeae gained from sequencing may translate into the identification or development of new therapeutic agents.

\section{S12 - Sexual health issues for Indigenous youth in Australia and New Zealand}

Indigenous peoples worldwide suffer disproportionately poor sexual and reproductive health. This symposium will describe the sexual and reproductive health issues for Indigenous youth in Australia and New Zealand. Given that both Indigenous populations are demographically young in age compared to other ethnic groups, they require strategies that address their unique developmental and cultural needs. We will draw on data from 